\title{
Shadowgraph Imaging of Cavitating Jet
}

\author{
Ryuta Watanabe1, Takayuki Kikuchi', Takayuki Yamagata ${ }^{2}$, Nobuyuki Fujisawa ${ }^{2}$ \\ ${ }^{1}$ Graduate School of Science and Technology, Niigata University, Niigata, Japan \\ ${ }^{2}$ Visualization Research Center, Niigata University, Niigata, Japan \\ Email: fujisawa@eng.niigata-u.ac.jp
}

Received 3 April 2015; accepted 15 May 2015; published 17 July 2015

Copyright (C) 2015 by authors and Scientific Research Publishing Inc.

This work is licensed under the Creative Commons Attribution International License (CC BY).

http://creativecommons.org/licenses/by/4.0/

c) (i) Open Access

\begin{abstract}
This paper deals with the statistical properties of unsteady structure of cavitating water-jet issuing into a stagnant fluid of water using the shadowgraph imaging combined with the proper orthogonal decomposition (POD) analysis. The experimental result indicates that the cavitating jet is composed of axisymmetric mode, while the periodic axial oscillation is found along the jet centerline. The reconstructed cavitation images show the presence of growing, shrinking and shedding motion in the cavitation cloud, which sustains a periodic behavior of the cavitating jet.
\end{abstract}

\section{Keywords}

\section{Cavitating Jet, POD Analysis, Shadowgraph, Periodic Structure}

\section{Introduction}

When the flow velocity of the submerged water-jet is high enough, the jet accompanies the cavitation bubbles in the shear layer. Such flow field is called cavitating jet and has been in keen interests in engineering field for fabricating and processing the metal materials. Therefore, the cavitating jet has been a topic of interests both from the fundamental standpoint as well as for application to industrial field [1].

There are many studies on the visual observation of the cavitating jet in literature [2]-[7]. The flow observations are carried out using the shadowgraph technique. This is effective in combination with the high-speed imaging, which enables the time series observation of the instantaneous behavior of the cavitating jet. The observation reveals that the cavitation cloud is periodically generated in the nozzle by the self-resonance of the flow and the cloud-like cavitating structure is discharged from the nozzle periodically. The high-speed observation of the cavitation phenomenon inside the nozzle shows that the periodic structure accompanying the cavitation bubbles is generated in the shear layer and the collapse of the bubbles results in the cavitation erosion of metal materials [2] [6] [7]. It is known that the periodic structure of the cavitating jet is due to the reentrant motion in the nozzle. Therefore, the studies on the flow behavior inside the nozzle have been the topics of interests to understand the 
physical mechanism of the cavitating jet downstream of the nozzle. More recently, it is found that the performance of the cavitation erosion is amplified by increasing the self-resonance of the reentrant motion in the nozzle, so that the nozzle geometry is important to magnify the cavitation erosion [6]. Although there are many studies on the flow visualization of cavitating jet, the statistical analysis has not been introduced into the cavitating jet research.

Proper orthogonal decomposition (POD) is one of the statistical methods for analyzing the low-dimensional representation of multi-dimensional flow field of interest. The snapshot POD is very useful for recognizing the coherent structure of turbulent flow [8] [9], channel flow [10], flow around a square cylinder [11], flame flickering [12] and highly buoyant jet [13]. By introducing the POD analysis, most energetic structure of the flow is extracted by decomposing the fluctuating properties of the fluid into the linear sum of orthogonal eigenfunctions of temporal and spatial correlations.

The purpose of this paper is to introduce the snapshot POD analysis for understanding the statistical properties of cavitating jet behavior observed by shadowgraph imaging.

\section{Experimental Apparatus and Methods}

The experiments were carried out using an enclosure of square horizontal cross-sectional area of $400 \mathrm{~mm} \times$ $400 \mathrm{~mm}$ with a height of $400 \mathrm{~mm}$, which is illustrated in Figure 1(a). The cavitating jet of water issuing from a nozzle is injected into a water environment. The working fluid water is kept at temperature 20 degrees centigrade. Note that the nozzleis connected to a plunger pump of maximum operating pressure of 13 MPa. The details of the nozzle are shown in Figure 1(b), which is designed in reference to the study of cavitating jet to maximize the cavitation erosion [6]. It consists of two orifice plates of different diameters 3 $\mathrm{mm}$ and $0.8 \mathrm{~mm}$ with a separating distance of $6 \mathrm{~mm}$. Each orifice plate has a thickness of $3 \mathrm{~mm}$. The nozzle pressure is kept to $8 \mathrm{MPa}$ and is located at $50 \mathrm{~mm}$ deep from the water surface, so that the cavitation coefficient is 0.012 . The jet velocity at the nozzle exit is expected to be $100 \mathrm{~m} / \mathrm{s}$, which is obtained from the discharge coefficient of the nozzle 0.8 and the Bernoulli's principle. The flow visualization of the cavitating jet was conducted by the shadowgraph imaging and the images of cavitating jet were captured by a high-speed CMOS camera (1016 × 1016 pixels with 8 bits) operating at frame rate of $6000 \mathrm{frame} / \mathrm{sec}$ with an exposure time of $8 \mu \mathrm{sec}$. Total 2000 images were captured for the statistical analysis of the shadowgraph images. The target area of the image is $45 \mathrm{~mm}$ in axial direction from the nozzle exit and $20 \mathrm{~mm}$ in the normal direction. An illumination was provided from a halogen lamp, which is placed opposite side of the camera. It should be mentioned that the ground glass was installed between the lamp and the test section to make the illumination uniform. The lateral and axial coordinates $x, y$ with respect to the jet direction are taken as shown in Figure 1, where the origin is located at the exit of the nozzle.

\section{Snapshot POD Analysis}

The snapshot POD analysis is introduced into the image analysis of 2000 instantaneous shadowgraph images

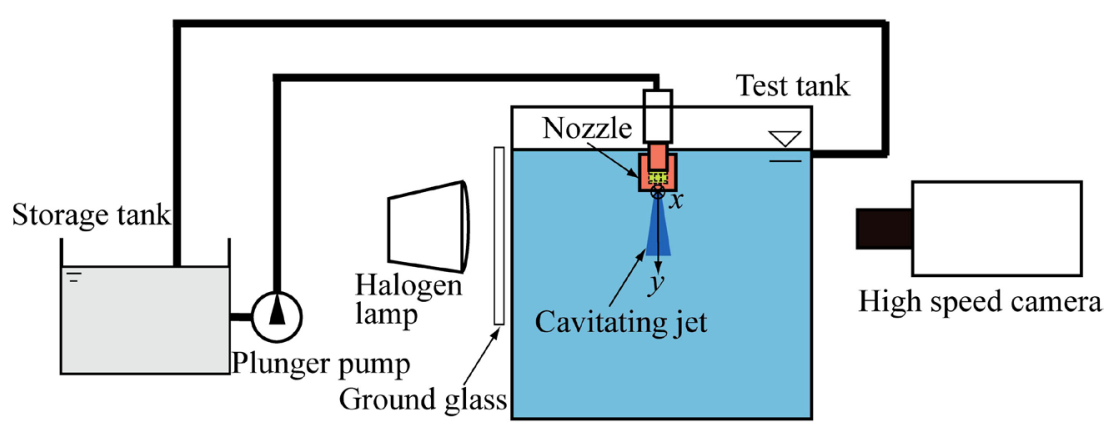

(a)

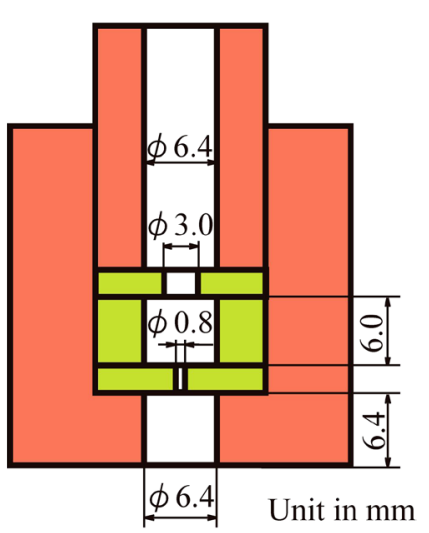

(b)

Figure 1. Experimental setup and nozzle. (a) Experimental setup; (b) Nozzle geometry (Orifice thickness $3 \mathrm{~mm}$ ). 
taken by a CMOS camera operating at $6000 \mathrm{~Hz}$. The basic idea of snapshot POD is that it yields a set of orthogonal eigenfunctions that are optimal in energy representing temporal and spatial correlations of instantaneous shadowgraph images. The POD analysis is conducted on 2000 snapshots of instantaneous shadowgraph images. The sufficiency of the number of images is confirmed by reducing the number of images to the half. The analysis allows the evaluation of mean (0th mode) and fluctuating properties (1st mode, 2nd mode, ---) of the shadowgraph images, so that the result indicates the statistical properties of the cavitating jet. The details of the snapshot POD analysis have been described elsewhere [13], so that it is not repeated in this paper.

\section{Results and Discussions}

\subsection{Time Series Shadowgraph Images}

Figure 2 shows time-series shadowgraph images taken by a CMOS camera operating at 6000 frames/sec. Note that the nozzle pressure is set to $8 \mathrm{MPa}$, while the images are shown for every $0.17 \mathrm{msec}$. in time interval. These images show the unsteady behavior of the cavitating jet, such as the growth of the cavitation cloud downstream of the nozzle followed by shrinking motion due to the reentrant behavior and the shedding. Such periodic behavior of the cavitating jet has been observed by Sato et al. [5] in a convergent-divergent nozzle due to the self-resonance behavior in the nozzle. It should be mentioned that a periodic frequency was found to be $1700 \mathrm{~Hz}$ in the present experiment, which is obtained from the FFT analysis of the shadowgraph images.

\subsection{POD Analysis}

Figure 3 shows the first four POD modes of the shadowgraph images of the present cavitating jet issuing from an orifice-type nozzle in the nozzle pressure of $8 \mathrm{MPa}$. It should be mentioned that the 0th POD mode (a) shows the mean contour of cavitating jet, which distributes axisymmetrically along the centerline. It is seen that the average length of the cavitation is about $15 \mathrm{~mm}$ from the nozzle. On the other hand, 1st (b), 2nd (c) and 3rd mode (d) indicate the fluctuating properties of the cavitating jet. The positive peak appears in the 1st mode along the shear layer near the nozzle and the negative peak is found further downstream. This result indicates that the fluctuation of the cavitation cloud is axisymmetric with respect to the jet centerline, while the axial fluctuation is periodic due to the growing motion in the positive peak and shrinking motion in the negative peak downstream, which is followed by the weak positive region further downstream. Similarly, the positive and negative peaks appear in the 2nd mode along the jet shear layer. They are located between the peaks in the 1st POD mode. It should be mentioned that each energy of the 1 st POD mode is $19 \%$, 2nd mode is $15 \%$ and 3rd mode is $4 \%$, so that $1 / 3$ of the total energy is involved in the first 2 fluctuating POD modes. These results indicate that the cavitating jet shows the periodic axial oscillation from the statistical point of view, which corresponds to the growing, shrinking and shedding motion of the cavitating jet. This result agrees closely with the observation of

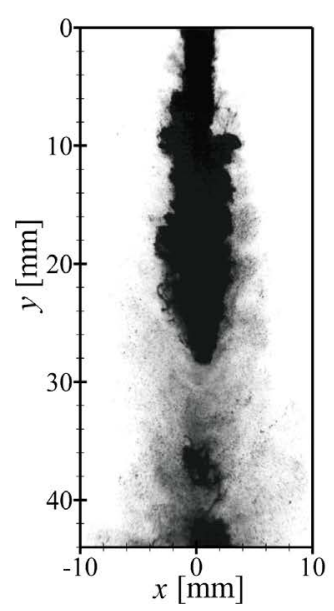

(a)

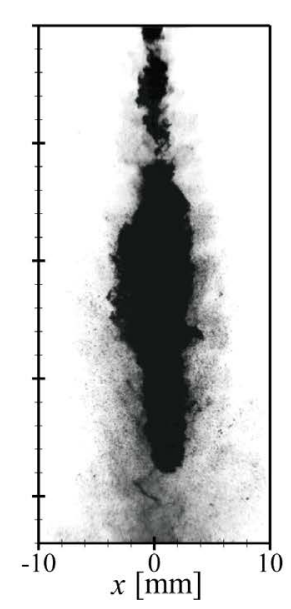

(b)

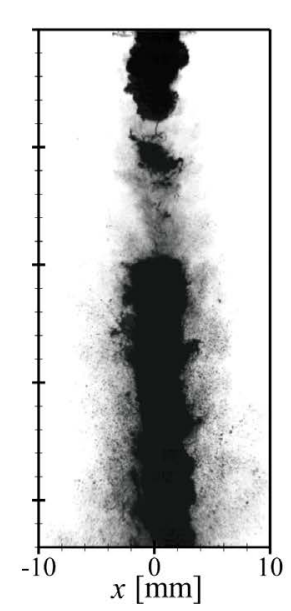

(c)

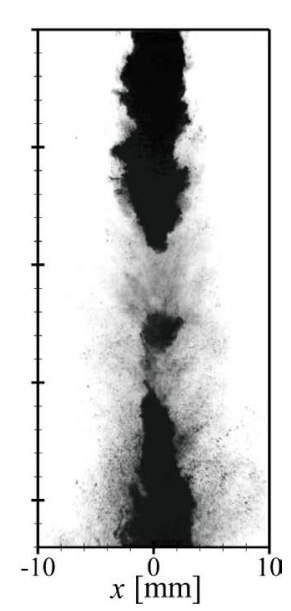

(d)

Figure 2. Shadowgraph images of cavitating jet. (a) $t=0 \mathrm{sec}$; (b) $t=0.17 \mathrm{msec}$; (c) $t=0.33$ msec; (d) $t=0.5$ msec. 
instantaneous shadowgraph images in Figure 2.

Figure 4 shows a periodic motion of the cavitating jet reconstructed from the 0th POD mode and the first 10 fluctuating POD modes. The reconstruction was carried out at the same instants of the shadowgraph images shown in Figure 2. The fluctuating energy of the 10 modes covers $50 \%$ of the total energy. The reconstructed images of the cavitating jet indicate the fundamental cavitating jet behavior by neglecting the higher POD modes. They show the periodical variation of the cavitation cloud, which corresponds to the growing, shrinking and the shedding motion of the cavitating jet, although the original shadowgraph images are noisy due to the unsteady behavior of turbulence in the jet shear layer. These results indicate that the POD analysis provides a statistical basis for understanding the unsteady behavior of the cavitating jet, which also supports the periodic motion of the cavitating jet suggested in literature [2] [4]-[7].

\section{Conclusion}

The unsteady structure of the cavitating jet from an orifice-type nozzle issuing into stagnant fluid of water is

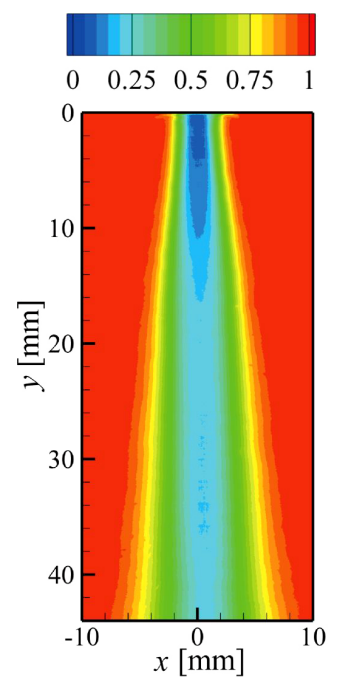

(a)

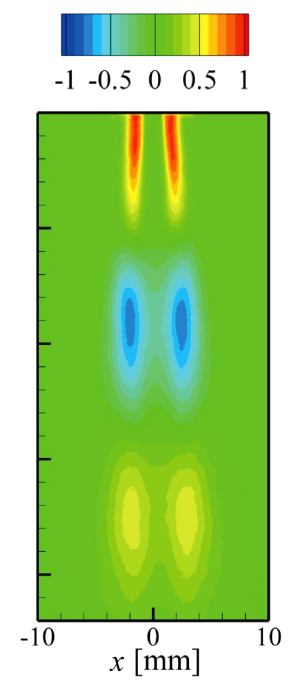

(b)

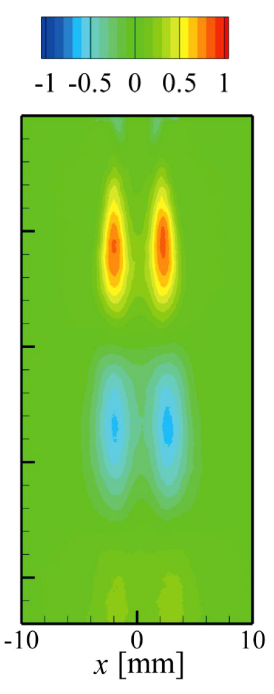

(c)

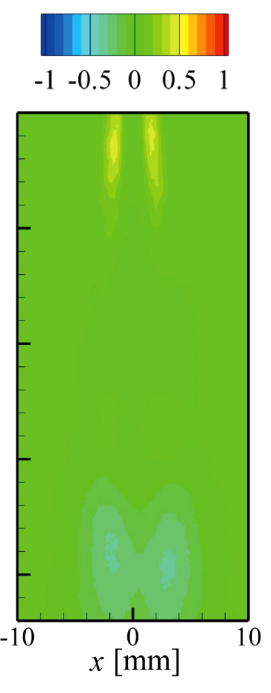

(d)

Figure 3. POD modes of cavitating jet. (a) 0th mode; (b) 1st mode; (c) 2nd mode; (d) 3rd mode.

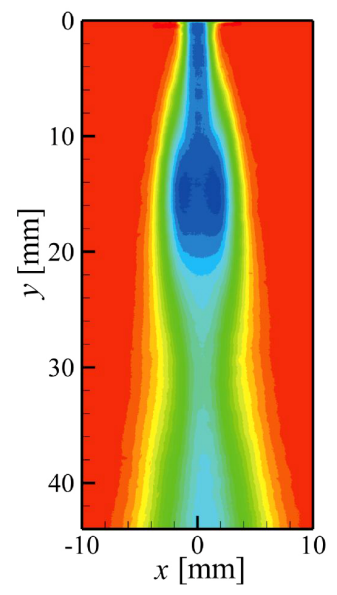

(a)

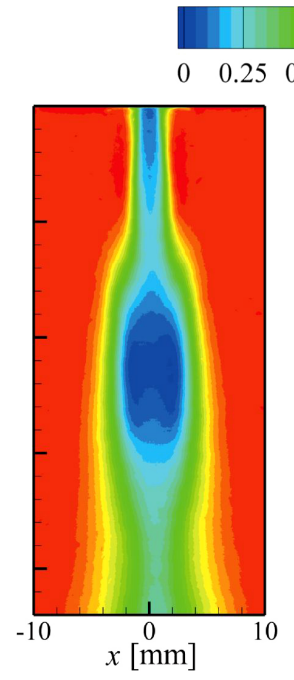

(b)
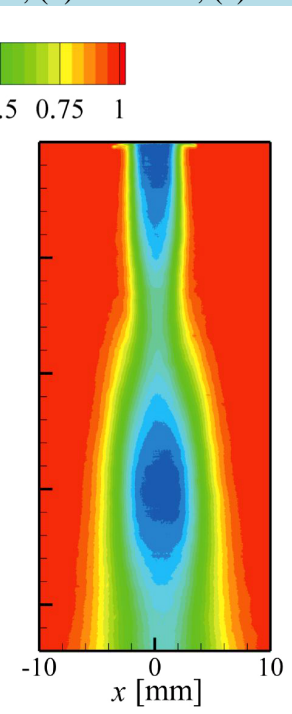

(c)

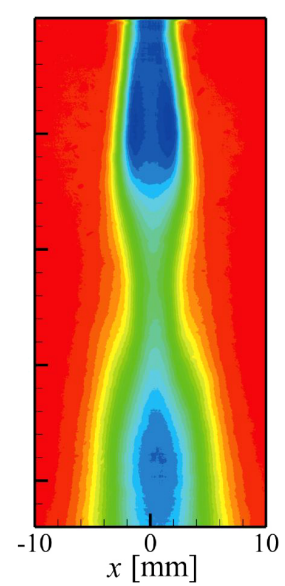

(d)

Figure 4. Reconstruction of a cycle of reentrant motion of cavitating jet. (a) $t=0 \mathrm{sec}$; (b) $t=0.17$ msec; (c) $t=0.33$ msec; (d) $t=0.5$ msec. 
studied experimentally using the shadowgraph imaging in combination with the proper orthogonal decomposition (POD) analysis. The statistical result indicates that the behavior of the cavitation cloud is axisymmetric with respect to the jet axis, while the cavitation cloud oscillates periodically in the axial direction and the appearance and the disappearance of the cavitation cloud is observed periodically in the downstream. This phenomenon corresponds to the growing, shrinking and shedding motion of the cavitating jet observed in the raw shadowgraph images.

\section{Acknowledgements}

The authors thank to Prof. T. Kagawa from Tokyo Institute of Technology and the members of cavitation research group in The Japan Fluid Power System Society for the helpful comments on this work.

\section{References}

[1] Franc, J.P. and Michel, J.M. (2004) Fundamentals of Cavitation, Kluwer Academic Publishers, Kluwer.

[2] Soyama, H., Yanauchi, Y., Sato, K., Ikohagi, T., Oba, R. and Oshima, R. (1996) High-Speed Observation of Ultrahigh-Speed Submerged Water Jets. Experimental Thermal and Fluid Science, 12, 411-416. http://dx.doi.org/10.1016/0894-1777(95)00124-7

[3] Sato, K. and Saito, Y. (2002) Unstable Cavitation Behavior in a Circular-Cylindrical Orifice Flow. JSME International Journal, Series B, 45, 638-645. http://dx.doi.org/10.1299/jsmeb.45.638

[4] Hutli, E.A.F. and Nedeljkovic, M.S. (2008) Frequency in Shedding/Discharging Cavitation Cloud Determined by Visualization of a Submerged Cavitating Jet. Journal of Fluids Engineering, 130, Article ID: 021304.

[5] Sato, K., Taguchi, Y. and Hayashi, S. (2013) High Speed Observation of Periodic Behavior in a Convergent-Divergent Nozzle for Cavitating Water Jet. Journal of Flow Control, Measurement and Visualization, 1, 102-107. http://dx.doi.org/10.4236/jfcmv.2013.13013

[6] Soyama, H. (2014) Enhancing the Aggressive Intensity of a Cavitating Jet by Introducing a Cavitator and a Guide Pipe. Journal of Fluid Science and Technology, 9, 1-12.

[7] Stanley, C., Barber, T. and Rosengarten, G. (2014) Re-Entrant Jet Mechanism for Periodic Cavitation Shedding in a Cylindrical Orifice. International Journal of Heat and Fluid Flow, 50, 169-176. http://dx.doi.org/10.1016/j.ijheatfluidflow.2014.07.004

[8] Sirovich, L. (1987) Turbulence and the Dynamics of Coherent Structures, Part 1: Coherent Structures. Quarterly of Applied Mathematics, 45, 561-571.

[9] Berkooz, G., Holmes, P. and Lumley, J.L. (1993) The Proper Orthogonal Decomposition in the Analysis of Turbulent Flows. Annual Review of Fluid Mechanics, 25, 539-575. http://dx.doi.org/10.1146/annurev.fl.25.010193.002543

[10] Liu, Z.-C., Adrian, R.J. and Hanratty, T.J. (2001) Large-Scale Modes of Turbulent Channel Flow, Transport and Structure. Journal of Fluid Mechanics, 448, 53-80.

[11] van Oudheusden, B.W., Scarano, F., van Hinsberg, N.P. and Watt, D.W. (2005) Phase-Resolved Characteristics of Vortex Shedding in the Near Wake of a Square-Section Cylinder at Incidence. Experiments in Fluids, 39, 86-98. http://dx.doi.org/10.1007/s00348-005-0985-5

[12] Fujisawa, N., Yamada, J. and Yamagata, T. (2014) Measurement of Three-Dimensional Temperature Field of Flickering Premixed Flame with and without Co-Flow. Flow, Turbulence and Combustion, 93, 723-739. http://dx.doi.org/10.1007/s10494-014-9568-y

[13] Watanabe, R., Yamagata, T. and Fujisawa, N. (2015) Three-Dimensional Flow Structure in Highly Buoyant Jet by Scanning Stereo PIV Combined with POD Analysis. International Journal of Heat and Fluid Flow, 52, 98-110. http://dx.doi.org/10.1016/j.ijheatfluidflow.2014.12.003 